\title{
The Series of Reciprocals of Non-central Binomial Coefficients
}

\author{
Laiping Zhang, Wanhui Ji \\ Yinchan Energy College, Yinchuan, China \\ Email: zhanglaiping79@163.com, jiwanhui2008@163.com
}

Received 2013

\begin{abstract}
Utilizing Gamma-Beta function, we can build one series involving reciprocal of non-central binomial coefficients, then We can structure several new series of reciprocals of non-central binomial coefficients by item splitting, these new created denominator of series contain 1 to 4 odd factors of binomial coefficients. As the result of splitting items, some identities of series of numbers values of reciprocals of binomial coefficients are given. The method of splitting terms offered in this paper is a new combinatorial analysis way and elementary method to create new series.
\end{abstract}

Keywords: Binomial Coefficients; Split Terms; Reciprocals; Series; Non-central; Closed Form

\section{Introduction}

Binomial coefficient series plays an important role among Number theory, Graph Theory, mathematical statistics and Probability Theory, etc mathematics branches. Binomial coefficient series conversion also plays key role in research areas of combinatorial mathematics and mathematical analysis, it aroused great attention and can be referred to a large number of literature [1-7]. Paper [2-5] the author used is called Lehmer series identity

$$
\sum_{n=1}^{\infty} \frac{(2 x)^{2 n}}{n\left(\begin{array}{c}
2 n \\
n
\end{array}\right)}=\frac{2 x \arcsin x}{\sqrt{1-x^{2}}},|x|<1,
$$

Some authors differential, integral, generating function, the white to Beta-gamma function, hyper geometric function series, recurrence and other mathematical tools to get important results on series of reciprocals of binomial coefficients. Paper [8] obtains alternating series involving reciprocals of central binomial coefficients. Paper [9] obtains series involving reciprocals of central binomial coefficients. We did the research the series of reciprocal of non-central binomial coefficient. Utilizing Gamma-beta function, we obtain one series involving reciprocal of non-central binomial coefficients. We can structure several new series of reciprocal of non-central binomial coefficients by splitting item; the new created denominators of series contain 1 to 4 odd factors of non-central binomial coefficients.

By continuously using the method of splitting items, we can get the series involving reciprocal of non-central.

Binomial coefficients of which denominator contain 5odd factor, 6 odd factor, ... and p odd factor.
Therefore splitting term method in this paper is an elementary to build new series and also provide a new combinatorial analysis method. As the result of analysis some identities of series of number values of reciprocal of non-central binomial coefficients also provided in this paper.

\section{Main Results and Proof of Theorem}

Theorem 1 one series of reciprocals of non-central binomial coefficients

$$
\begin{aligned}
& \sum_{k=0}^{\infty} \frac{x^{k}}{\left(\begin{array}{c}
2 k+1 \\
k
\end{array}\right)}=D \\
& =\left\{\begin{array}{l}
\frac{2}{4-x}+\frac{8}{(4-x) \sqrt{4 x-x^{2}}} \arctan \frac{x}{\sqrt{4 x-x^{2}}}, 0<x<4 \\
\frac{2}{4-x}+\frac{4}{(4-x) \sqrt{-4 x+x^{2}}} \ln \frac{\sqrt{-4 x+x^{2}}-x}{\sqrt{-4 x+x^{2}}+x},-4<x<0
\end{array}\right.
\end{aligned}
$$

Proof From power series theories easy to understand, the series

$$
\sum_{k=0}^{\infty} \frac{x^{k}}{\left(\begin{array}{c}
2 k+1 \\
k
\end{array}\right)}=\sum_{k=0}^{\infty} \frac{x^{k}}{\left(\begin{array}{c}
2 k+1 \\
k+1
\end{array}\right)}
$$

is convergence.

Over interval $(-4,4)$. Utilizing Gamma-Beta function relation and binomial coefficients,

$$
\left(\begin{array}{l}
n \\
k
\end{array}\right)^{-1}=(n+1) \int_{0}^{1} t^{k}(1-t)^{n-k} d t
$$


we calculation sum function of series

$$
\begin{aligned}
& \sum_{k=0}^{\infty} \frac{x^{k}}{\left(\begin{array}{c}
2 k+1 \\
k
\end{array}\right)}=\sum_{k=0}^{\infty} x^{k}(2 k+2) \int_{0}^{1} t^{k+1}(1-t)^{k} d t \\
= & 2 \int_{0}^{1} \sum_{k=0}^{\infty} k x^{k} t^{k+1}(1-t)^{k} d t+2 \int_{0}^{1} \sum_{k=0}^{\infty} x^{k} t^{k+1}(1-t)^{k} d t \\
= & 2 \int_{0}^{1} \sum_{k=0}^{\infty} k[x t(1-t)]^{k} t d t+2 \int_{0}^{1} \sum_{k=0}^{\infty}[x t(1-t)]^{k} t d t^{\prime} \\
= & 2 \int_{0}^{1} \frac{x t(1-t)}{[1-x t(1-t)]^{2}} d t+2 \int_{0}^{1} \frac{t d t}{1-x t(1-t)} \\
= & -2 \int_{0}^{1} \frac{1-x t(1-t)+1}{[1-x t(1-t)]^{2}} t d t+2 \int_{0}^{1} \frac{t d t}{1-x t(1-t)} \\
= & 2 \int_{0}^{1} \frac{t d t}{\left[1-x t+x t^{2}\right]^{2}}
\end{aligned}
$$

Using recursion formulas of integral [7]

$$
\begin{aligned}
& =2\left[\frac{1}{4-x}+\frac{1}{4-x} \int_{0}^{1} \frac{1}{1-x t+x t^{2}} d t\right] \\
& =\frac{2}{4-x}+\frac{2}{4-x} \int_{0}^{1} \frac{1}{1+x t-x t^{2}} d t \\
& =\frac{2}{4-x}+\frac{8}{(4-x) \sqrt{4 x-x^{2}}} \arctan \frac{x}{\sqrt{4 x-x^{2}}},
\end{aligned}
$$

for $0<x<4$

$$
=\frac{2}{4-x}+\frac{4}{(4-x) \sqrt{-4 x+x^{2}}} \ln \frac{\sqrt{-4 x+x^{2}}-x}{\sqrt{-4 x+x^{2}+x}},
$$

for $-4<x<0$. This completes proof of Theorem 1 .

Theorem 2 The series of reciprocals of non-central binomial coefficients

1) The denominator contains 1 odd factor of binomial coefficients in the series

$$
\begin{aligned}
& \sum_{m=0}^{\infty} \frac{x^{m}}{\left(\begin{array}{c}
2 m+1 \\
m
\end{array}\right)(2 m+3)}=\left(\frac{4}{x}-1\right) D-\frac{4}{x} \\
& \sum_{m=0}^{\infty} \frac{x^{m}}{\left(\begin{array}{c}
2 m+1 \\
m
\end{array}\right)(2 m+5)}=\left(\frac{32}{x^{2}}-\frac{12}{x}+1\right) D-\frac{32}{x^{2}}+\frac{4}{3 x} \\
& \sum_{m=0}^{\infty} \frac{x^{m}}{\left(\begin{array}{c}
2 m+1 \\
m
\end{array}\right)(2 m+7)}=\left(\frac{512}{3 x^{3}}-\frac{64}{x^{2}}+\frac{4}{x}+\frac{1}{3}\right) \\
& \times D-\frac{512}{3 x^{3}}+\frac{64}{9 x^{2}}+\frac{4}{15 x} \\
& \sum_{m=0}^{\infty} \frac{x^{m}}{\left(\begin{array}{c}
2 m+1 \\
m
\end{array}\right)(2 m+9)}=\left(\frac{4096}{5 x^{4}}-\frac{1536}{5 x^{3}}+\frac{96}{5 x^{2}}+\frac{4}{5 x}+\frac{1}{5}\right) \\
& \times D-\frac{4096}{5 x^{4}}+\frac{512}{15 x^{3}}+\frac{32}{25 x^{2}}+\frac{4}{35 x}
\end{aligned}
$$

2) The denominator contains 2 odd factors of binomial coefficients in the series

$$
\begin{aligned}
& \sum_{m=0}^{\infty} \frac{x^{m}}{\left(\begin{array}{c}
2 m+1 \\
m
\end{array}\right)(2 m+3)(2 m+5)} \\
&=\left(-\frac{16}{x^{2}}+\frac{8}{x}-1\right) D+\frac{16}{x^{2}}-\frac{8}{3 x} \\
& \sum_{m=0}^{\infty} \frac{x^{m}}{\left(\begin{array}{c}
2 m+1 \\
m
\end{array}\right)(2 m+3)(2 m+7)} \\
&=\left(-\frac{128}{3 x^{3}}+\frac{16}{x^{2}}-\frac{1}{3}\right) D+\frac{128}{3 x^{3}}-\frac{16}{9 x^{2}}-\frac{16}{15 x} \\
& \sum_{m=0}^{\infty} \frac{\left(\begin{array}{c}
2 m+1 \\
m
\end{array}\right)(2 m+5)(2 m+7)}{3 x^{m}} \\
&=\left(-\frac{256}{3 x^{3}}+\frac{48}{x^{2}}-\frac{8}{x}+\frac{1}{3}\right) D+\frac{256}{3 x^{3}}-\frac{176}{9 x^{2}}+\frac{8}{15 x} \\
& \sum_{m=0}^{\infty} \frac{x^{m}}{\left(\begin{array}{c}
2 m+1 \\
m
\end{array}\right)(2 m+3)(2 m+9)} \\
&=\left(-\frac{2048}{15 x^{4}}+\frac{256}{5 x^{3}}-\frac{16}{5 x^{2}}+\frac{8}{15 x}-\frac{1}{5}\right) D \\
&+\frac{2048}{15 x^{4}}-\frac{256}{45 x^{3}}-\frac{16}{75 x^{2}}-\frac{24}{35 x} \\
& \sum_{m=0}^{\infty} \frac{x^{m}}{\left(\begin{array}{l}
2 m+1 \\
m
\end{array}\right)(2 m+5)(2 m+9)} \\
&=\left(-\frac{1024}{5 x^{4}}+\frac{384}{5 x^{3}}+\frac{16}{5 x^{2}}-\frac{16}{5 x}+\frac{1}{5}\right) D \\
&+\frac{1024}{5 x^{4}}-\frac{128}{15 x^{3}}-\frac{208}{25 x^{2}}+\frac{32}{105 x} \\
&\left.\frac{2048}{5 x^{4}}+\frac{3584}{15 x^{3}}-\frac{208}{5 x^{2}}+\frac{8}{5 x}+\frac{512}{5 x^{3}}+\frac{656}{225 x^{2}}+\frac{8}{15}\right) D \\
& \\
&
\end{aligned}
$$

3) The denominator contains 3 odd factors of binomial coefficients in the series

$$
\begin{aligned}
& \sum_{m=0}^{\infty} \frac{x^{m}}{\left(\begin{array}{c}
2 m+1 \\
m
\end{array}\right)(2 m+3)(2 m+5)(2 m+7)} \\
= & \left(\frac{64}{3 x^{3}}-\frac{16}{x^{2}}+\frac{4}{x}-\frac{1}{3}\right) D-\frac{64}{3 x^{3}}+\frac{80}{9 x^{2}}-\frac{4}{5 x} \\
& \sum_{m=0}^{\infty} \frac{x^{m}}{\left(\begin{array}{c}
2 m+1 \\
m
\end{array}\right)(2 m+3)(2 m+5)(2 m+9)} \\
= & \left(\frac{512}{15 x^{4}}-\frac{64}{5 x^{3}}-\frac{16}{5 x^{2}}+\frac{28}{15 x}-\frac{1}{5}\right) D \\
& -\frac{512}{15 x^{4}}+\frac{64}{45 x^{3}}+\frac{304}{75 x^{2}}-\frac{12}{105 x}
\end{aligned}
$$




$$
\begin{aligned}
& \sum_{m=0}^{\infty} \frac{x^{m}}{\left(\begin{array}{c}
2 m+1 \\
m
\end{array}\right)(2 m+3)(2 m+7)(2 m+9)} \\
= & \left(\frac{1024}{15 x^{4}}-\frac{704}{15 x^{3}}+\frac{48}{5 x^{2}}-\frac{4}{15 x}-\frac{1}{15}\right) D \\
& -\frac{1024}{15 x^{4}}+\frac{1088}{45 x^{3}}-\frac{176}{225 x^{2}}-\frac{4}{21 x} \\
& \sum_{m=0}^{\infty} \frac{x^{m}}{\left(\begin{array}{c}
m \\
m
\end{array}\right)(2 m+5)(2 m+7)(2 m+9)} \\
= & \left(\frac{512}{5 x^{4}}-\frac{1216}{15 x^{3}}+\frac{112}{5 x^{2}}-\frac{12}{5 x}+\frac{1}{15}\right) D \\
& -\frac{512}{5 x^{4}}+\frac{704}{15 x^{3}}-\frac{1228}{225 x^{2}}+\frac{4}{35 x}
\end{aligned}
$$

4) The denominator contains 4 odd factors of binomial coefficients in the series

$$
\begin{aligned}
& \sum_{m=0}^{\infty} \frac{x^{m}}{\left(\begin{array}{c}
2 m+1 \\
m
\end{array}\right)(2 m+3)(2 m+5)(2 m+7)(2 m+9)} \\
= & \left(-\frac{256}{15 x^{4}}+\frac{256}{15 x^{3}}-\frac{32}{5 x^{2}}+\frac{16}{15 x}-\frac{1}{15}\right) D \\
& +\frac{256}{15 x^{4}}-\frac{512}{45 x^{3}}+\frac{544}{225 x^{2}}-\frac{16}{105 x}
\end{aligned}
$$

\section{Proof of Theorem}

1) Left sides of (0) splitting terms

$$
\begin{gathered}
\sum_{n=0}^{\infty} \frac{n !(n+1) ! x^{n}}{(2 n+1) !}=D \\
\sum_{n=0}^{\infty} \frac{n !(n+1) ! x^{n}}{(2 n+1) !} \\
=1+\sum_{n=0}^{\infty} \frac{(n-1) ! n !(n)(n+1) x^{n}}{(2 n-2+1) !(2 n-1+1)(2 n+1)}=D,
\end{gathered}
$$

put $n-1=m$,

$$
1+\sum_{m=0}^{\infty} \frac{m !(m+1) !(m+1)(m+2) x^{m+1}}{(2 m+1) !(2 m+2)(2 m+3)}=D,
$$

Multiply both sides by $\frac{4}{x}$, arrive to

$$
\begin{aligned}
& \frac{4}{x}+\sum_{m=0}^{\infty} \frac{m !(m+1) !(2 m+3+1) x^{m}}{(2 m+1) !(2 m+3)}=\frac{4}{x} D, \\
& \frac{4}{x}+\sum_{m=0}^{\infty} \frac{m !(m+1) ! x^{m}}{(2 m+1) !}\left(1+\frac{1}{2 m+3}\right)=\frac{4}{x} D,
\end{aligned}
$$

We obtain (1). Let right sides (1) be $D_{3} .(2)$. Left sides of (0) splitting terms

$$
\begin{aligned}
& 1+\frac{x}{3} \\
& +\sum_{n=0}^{\infty} \frac{(n-2) !(n-1) !(n-1) n \bullet n(n+1) x^{n}}{(2 n-4+1) !(2 n-3+1)(2 n-2+1)(2 n-1+1)((2 n+1)} \\
& =D \text {, } \\
& \text { put } \quad n-2=m \\
& \qquad \begin{array}{l}
3+\frac{x}{3} \\
\quad+\sum_{m=0}^{\infty} \frac{m !(m+1) !(m+1)(m+2)(m+2)(m+3) x^{m+2}}{(2 m+1) !(2 m+2)(2 m+3)(2 m+4)(2 m+5)} \\
=D,
\end{array}
\end{aligned}
$$

Multiply both sides by $\frac{16}{x^{2}}$, arrive to

$$
\begin{aligned}
& \frac{16}{x^{2}}+\frac{16}{3 x}+\sum_{m=0}^{\infty} \frac{m !(m+1) ! x^{m}}{(2 m+1) !} \\
& \times\left(1+\frac{1}{2 m+3}\right)\left(1+\frac{1}{2 m+5}\right)=\frac{16}{x^{2}} D \\
& \frac{16}{x^{2}}+\frac{16}{3 x}+\sum_{m=0}^{\infty} \frac{m !(m+1) ! x^{m}}{(2 m+1) !} \\
& \times\left(1+\frac{1}{2 m+3}+\frac{1}{2 m+5}+\frac{1}{(2 m+3)(2 m+5)}\right)=\frac{16}{x^{2}} D \\
& \frac{16}{x^{2}}+\frac{16}{3 x}+\sum_{m=0}^{\infty} \frac{m !(m+1) ! x^{m}}{(2 m+1) !} \\
& \times\left(1+\frac{3 / 2}{2 m+3}+\frac{1 / 2}{2 m+5}\right)=\frac{16}{x^{2}} D
\end{aligned}
$$

Multiply both sides by 2 , arrive to

$$
\begin{aligned}
& \frac{32}{x^{2}}+\frac{32}{3 x}+2 D+3 D_{3} \\
& +\sum_{m=0}^{\infty} \frac{m !(m+1) ! x^{m}}{(2 m+1) !(2 m+5)}=\frac{32}{x^{2}} D
\end{aligned}
$$

We obtain (2). Let right sides (2) be $D_{5}$. 3) Left sides of (0) splitting terms

$$
\begin{aligned}
& 1+\frac{x}{3}+\frac{x^{2}}{10} \\
& +\sum_{n=0}^{\infty} \frac{(n-3) !(n-2) !(n-2)(n-1) n \cdot(n-1) n(n+1) x^{n}}{(2 n-6+1) !(2 n-5+1)(2 n-4+1) \cdots(2 n+1)} \\
& =D
\end{aligned}
$$

put $n-3=m$

$$
1+\frac{x}{3}+\frac{x^{2}}{10}+\sum_{m=0}^{\infty} \frac{m !(m+1) !(m+1)(m+2)(m+3)(m+2)(m+3)(m+4) x^{m+3}}{(2 m+1) !(2 m+2)(2 m+3)(2 m+4)(2 m+5)(2 m+6)(2 m+7)}=D
$$


arrive to

$$
\frac{256}{x^{4}}+\frac{256}{3 x^{3}}+\frac{128}{5 x^{2}}+\frac{256}{35 x}+\sum_{m=0}^{\infty} \frac{m !(m+1) ! x^{m}}{(2 m+1) !}\left[1+\frac{35 / 16}{2 m+3}+\frac{15 / 16}{2 m+5}+\frac{9 / 16}{2 m+7}+\frac{5 / 16}{2 m+9}\right]=\frac{256}{x^{4}} D
$$

Multiply both sides by $\frac{16}{5}$, arrive to

$$
\frac{4096}{5 x^{4}}+\frac{4096}{15 x^{3}}+\frac{2048}{25 x^{2}}+\frac{4096}{175 x}+\frac{16}{5} D+7 D_{3}+3 D_{5}+\frac{9}{5} D_{7}+\sum_{m=0}^{\infty} \frac{m !(m+1) ! x^{m}}{(2 m+1) !(2 m+9)}=\frac{4096}{5 x^{4}} D,
$$

We obtain (4), Let right sides (4) be $D_{9}$

2) In (0.1), the factions of 2 factors retained, other fractions divided partial fractions. Then spit term the fraction containing 2 factions, reserve one during each split term, what left has been split into partial fraction, arrive to

$$
\begin{array}{ll} 
& \frac{256}{x^{4}}+\frac{256}{3 x^{3}}+\frac{128}{5 x^{2}}+\frac{256}{35 x}+D_{3 \cdot 5}+D+\frac{27}{16} D_{3} \\
+ & \frac{23}{16} D_{5}+\frac{9}{16} D_{7}+\frac{5}{16} D_{9}=\frac{256}{x^{4}} D \\
\text { B) } & \frac{256}{x^{4}}+\frac{256}{3 x^{3}}+\frac{128}{5 x^{2}}+\frac{256}{35 x}+D_{3 \cdot 7}+D+\frac{31}{16} D_{3} \\
& +\frac{15}{16} D_{5}+\frac{13}{16} D_{7}+\frac{5}{16} D_{9}=\frac{256}{x^{4}} D \\
& \frac{256}{x^{4}}+\frac{256}{3 x^{3}}+\frac{128}{5 x^{2}}+\frac{256}{35 x}+D_{3 \cdot 9}+D+\frac{97}{48} D_{3} \\
& +\frac{15}{16} D_{5}+\frac{9}{16} D_{7}+\frac{23}{48} D_{9}=\frac{256}{x^{4}} D \\
\text { D) } & \frac{256}{x^{4}}+\frac{256}{3 x^{3}}+\frac{128}{5 x^{2}}+\frac{256}{35 x}+D_{5 \cdot 7}+D+\frac{35}{16} D_{3} \\
& +\frac{7}{16} D_{5}+\frac{17}{16} D_{7}+\frac{5}{16} D_{9}=\frac{256}{x^{4}} D . \\
& \frac{256}{x^{4}}+\frac{256}{3 x^{3}}+\frac{128}{5 x^{2}}+\frac{256}{35 x}+D_{5 \cdot 9}+D+\frac{35}{16} D_{3} \\
& +\frac{11}{16} D_{5}+\frac{9}{16} D_{7}+\frac{9}{16} D_{9}=\frac{256}{x^{4}} D . \\
& \frac{256}{x^{4}}+\frac{256}{3 x^{3}}+\frac{128}{5 x^{2}}+\frac{256}{35 x}+D_{7 \cdot 9}+D+\frac{35}{16} D_{3} \\
& +\frac{15}{16} D_{5}+\frac{1}{16} D_{7}+\frac{13}{16} D_{9}=\frac{1024}{x^{5}} D .
\end{array}
$$

Because $\mathrm{D}, D_{3}, D_{5}, D_{7}, D_{9}$ is known, the calculation of (A) - (F), we get (5) - (10),

3) In (0.1), the factions of 3 factors retained, other fractions divided partial fractions.

Then spit term the fraction containing 3 factions, reserve one during each split term, what left has been split into partial fraction, arrive to

$$
\text { A) } \frac{256}{x^{4}}+\frac{256}{3 x^{3}}+\frac{128}{5 x^{2}}+\frac{256}{35 x}+D_{3 \cdot 5 \cdot 7}+D+\frac{33}{16} D_{3}
$$

$$
+\frac{19}{16} D_{5}+\frac{7}{16} D_{7}+\frac{5}{16} D_{9}=\frac{256}{x^{4}} D .
$$

В) $\frac{256}{x^{4}}+\frac{256}{3 x^{3}}+\frac{128}{5 x^{2}}+\frac{256}{35 x}+D_{3 \cdot 5 \cdot 9}+D+\frac{101}{48} D_{3}$

B) $+\frac{17}{16} D_{5}+\frac{9}{16} D_{7}+\frac{13}{48} D_{9}=\frac{256}{x^{4}} D$.

C) $\frac{256}{x^{4}}+\frac{256}{3 x^{3}}+\frac{128}{5 x^{2}}+\frac{256}{35 x}+D_{3 \cdot 7 \cdot 9}+D+\frac{103}{48} D_{3}$ $+\frac{15}{16} D_{5}+\frac{11}{16} D_{7}+\frac{11}{48} D_{9}=\frac{256}{x^{4}} D$.

D) $\frac{256}{x^{4}}+\frac{256}{3 x^{3}}+\frac{128}{5 x^{2}}+\frac{256}{35 x}+D_{5 \cdot 7 \cdot 9}+D+\frac{35}{16} D_{3}$ $+\frac{13}{16} D_{5}+\frac{13}{16} D_{7}+\frac{3}{16} D_{9}=\frac{256}{x^{4}} D$.

Because $D, D_{3}, D_{5}, D_{7}, D_{9}$ is known, the calculation of (A) - (D), we get (11) - (14),

4) In (0.1), the factions of 4 factors retained, other fractions divided partial fractions.

Then spit term the fraction containing 4 factions, reserve one during each split term, what left has been split into partial fraction, arrive to

$$
\begin{aligned}
& \frac{256}{x^{4}}+\frac{256}{3 x^{3}}+\frac{128}{5 x^{2}}+\frac{256}{35 x}+D_{3 \cdot 5 \cdot 7 \cdot 9}+D+\frac{13}{6} D_{3} \\
& +D_{5}+\frac{1}{2} D_{7}+\frac{1}{3} D_{9}=\frac{256}{x^{4}} D .
\end{aligned}
$$

Because $D, D_{3}, D_{5}, D_{7}, D_{9}$, is known, the calculation of this expression, we get (15).

This completes proof of Theorem 2

\section{Some Series of Number Values}

In (1) - (15) of theorem 2, put $x=1$,

$$
D=\sum_{m=0}^{\infty} \frac{1}{\left(\begin{array}{c}
2 m+1 \\
m
\end{array}\right)}=\frac{2}{3}+\frac{4 \pi \sqrt{3}}{27}
$$

Put $x=-1$,

$$
D=\sum_{m=0}^{\infty} \frac{(-1)^{m}}{\left(\begin{array}{l}
2 m+1 \\
m
\end{array}\right)}=\frac{2}{5}+\frac{8 \sqrt{5}}{25} \ln \varphi, \quad \varphi=\frac{\sqrt{5}+1}{2} ;
$$

we have 
Corollary 1 The series of number values of reciprocal of non-central binomial coefficients
1) $\sum_{m=0}^{\infty} \frac{1}{\left(\begin{array}{l}2 m+1 \\ m\end{array}\right)(2 m+3)}=\frac{4 \pi \sqrt{3}}{9}-2$;
2) $\sum_{m=0}^{\infty} \frac{1}{\left(\begin{array}{c}2 m+1 \\ m\end{array}\right)(2 m+5)}=\frac{28 \pi \sqrt{3}}{9}-\frac{50}{3}$;
3) $\sum_{m=0}^{\infty} \frac{1}{\left(\begin{array}{c}2 m+1 \\ m\end{array}\right)(2 m+7)}=\frac{148 \pi \sqrt{3}}{9}-\frac{41058}{45}$;
4) $\sum_{m=0}^{\infty} \frac{1}{\left(\begin{array}{c}2 m+1 \\ m\end{array}\right)(2 m+9)}=\frac{2348 \pi \sqrt{3}}{9}-\frac{225158}{525}$;
5) $\sum_{m=0}^{\infty} \frac{1}{\left(\begin{array}{c}2 m+1 \\ m\end{array}\right)(2 m+3)(2 m+5)}=-\frac{4 \pi \sqrt{3}}{3}+\frac{22}{3}$;
6) $\sum_{m=0}^{\infty} \frac{1}{\left(\begin{array}{c}2 m+1 \\ m\end{array}\right)(2 m+3)(2 m+7)}=-4 \pi \sqrt{3}+\frac{1078}{45}$;
7) $\sum_{m=0}^{\infty} \frac{1}{\left(\begin{array}{c}2 m+1 \\ m\end{array}\right)(2 m+5)(2 m+7)}=-\frac{20 \pi \sqrt{3}}{3}+\frac{1634}{45}$;
8) $\sum_{m=0}^{\infty} \frac{1}{\left(\begin{array}{c}2 m+1 \\ m\end{array}\right)(2 m+3)(2 m+9)}=-\frac{196 \pi \sqrt{3}}{15}+\frac{112054}{1575}$;
9) $\sum_{m=0}^{\infty} \frac{1}{\left(\begin{array}{c}2 m+1 \\ m\end{array}\right)(2 m+5)(2 m+9)}=-\frac{284 \pi \sqrt{3}}{15}+\frac{18084}{175}$;
10) $\sum_{m=0}^{\infty} \frac{1}{\left(\begin{array}{c}2 m+1 \\ m\end{array}\right)(2 m+7)(2 m+9)}$
$=-\frac{156 \pi \sqrt{3}}{5}+\frac{267422}{1575}$
11)
$\sum_{m=0}^{\infty} \frac{1}{\left(\begin{array}{c}2 m+1 \\ m\end{array}\right)(2 m+3)(2 m+5)(2 m+7)}$
$=\frac{4 \pi \sqrt{3}}{3}-\frac{326}{45}$;
12)
$\sum_{m=0}^{\infty} \frac{1}{\left(\begin{array}{c}2 m+1 \\ m\end{array}\right)(2 m+3)(2 m+5)(2 m+9)}$
$=\frac{44 \pi \sqrt{3}}{15}-\frac{45316}{1575}$;
13)
$\sum_{m=0}^{\infty} \frac{1}{\left(\begin{array}{c}2 m+1 \\ m\end{array}\right)(2 m+3)(2 m+7)(2 m+9)}$
$=\frac{68 \pi \sqrt{3}}{15}-\frac{38842}{1575}$;
14)
$\sum_{m=0}^{\infty} \frac{1}{\left(\begin{array}{c}2 m+1 \\ m\end{array}\right)(2 m+5)(2 m+9)(2 m+9)}$
$=\frac{92 \pi \sqrt{3}}{15}-\frac{52306}{1575}$;

15)

$$
\begin{aligned}
& \sum_{m=0}^{\infty} \frac{1}{\left(\begin{array}{c}
2 m+1 \\
m
\end{array}\right)(2 m+3)(2 m+5)(2 m+7)(2 m+9)} \\
& =-\frac{4 \pi \sqrt{3}}{5}+\frac{762}{175} ;
\end{aligned}
$$

Corollary 2 Alternating series of number values of reciprocals of non-central binominal coefficients
1) $\sum_{m=0}^{\infty} \frac{(-1)^{m}}{\left(\begin{array}{l}2 m+1 \\ m\end{array}\right)(2 m+3)}=-\frac{8 \sqrt{5}}{5} \ln \varphi+2$;
2) $\sum_{m=0}^{\infty} \frac{(-1)^{m}}{\left(\begin{array}{c}2 m+1 \\ m\end{array}\right)(2 m+5)}=\frac{72 \sqrt{5}}{5} \ln \varphi-\frac{46}{3}$;
3) $\sum_{m=0}^{\infty} \frac{(-1)^{m}}{\left(\begin{array}{c}2 m+1 \\ m\end{array}\right)(2 m+7)}=-\frac{1144 \sqrt{5}}{15} \ln \varphi+\frac{3698}{45}$;
4) $\sum_{m=0}^{\infty} \frac{(-1)^{m}}{\left(\begin{array}{c}2 m+1 \\ m\end{array}\right)(2 m+9)}=\frac{1832 \sqrt{5}}{5} \ln \varphi-\frac{206938}{525}$;
5) $\sum_{m=0}^{\infty} \frac{(-1)^{m}}{\left(\begin{array}{c}2 m+1 \\ m\end{array}\right)(2 m+3)(2 m+5)}=-8 \sqrt{5} \ln \varphi+\frac{26}{3}$;
6) $\sum_{m=0}^{\infty} \frac{(-1)^{m}}{\left(\begin{array}{c}2 m+1 \\ m\end{array}\right)(2 m+3)(2 m+7)}=\frac{56 \sqrt{5}}{3} \ln \varphi-\frac{902}{45}$;
7) $\sum_{m=0}^{\infty} \frac{(-1)^{m}}{\left(\begin{array}{c}2 m+1 \\ m\end{array}\right)(2 m+5)(2 m+7)}=\frac{136 \sqrt{5}}{3} \ln \varphi-\frac{2194}{45}$;
8) $\sum_{m=0}^{\infty} \frac{(-1)^{m}}{\left(\begin{array}{c}2 m+1 \\ m\end{array}\right)(2 m+3)(2 m+9)}=-\frac{184 \sqrt{5}}{3} \ln \varphi+\frac{152294}{1575}$;
9) $\sum_{m=0}^{\infty} \frac{(-1)^{m}}{\left(\begin{array}{c}2 m+1 \\ m\end{array}\right)(2 m+5)(2 m+9)}=-88 \sqrt{5} \ln \varphi+\frac{16574}{175}$;
10) $\sum_{m=0}^{\infty} \frac{(-1)^{m}}{\left(\begin{array}{c}2 m+1 \\ m\end{array}\right)(2 m+7)(2 m+9)}=-\frac{664 \sqrt{5}}{3} \ln \varphi+\frac{375122}{1575}$;

\section{REFERENCES}

[1] B. Sury, T. N. Wang and F. Z. Zhao, "Some Identities Involving of Binomial Coefficients,” J. integer Sequences, Vol. 7, 2004,Article 04.2.8

[2] J. H. Yang and F. Z. Zhao, "Sums Involving the Inverses of Binomial Coefficients, Journal of Integer Sequences, Vol. 9, 2006, Article 06.4.2

[3] S. Amghibech, "On Sum Involving Binomial Coefficient,” Journal of Integer Sequences, Vol.10, 2007, Article 07.2.1

[4] T. Trif, "Combinatorial Sums and Series Involving Inverses of Binomial Coefficients,” Fibonacci Quarterly, Vol. 38, No. 1, 2000, pp. 79-84.

[5] F.-Z Zhao and T. Wang, "Some Results for Sums of the Inverses of Binomial Coefficients,” Integers: Electronic, Journal of combinatorial Number Theory, Vol. 5, No. 1, 
2005, p. A22.

[6] R. Sprugnoli, "Sums of Reciprocals of the Central Binomial Coefficients," Integers: Electronic Journal of Combinatorial Number Theory, Vol. 6 ,2006, p. A27

[7] I. S. Gradshteyn and I. M. Zyzhik, "A Table of Integral, Series and Products," Academic Press is an Imprint of Elsevier, Seventh Edition, Vol. 56, No. 61.

[8] W. H. Ji and L. P. Zhang, “On Series Alternated with
Positive and Negative Involving Reciprocals of Binominal Coefficients,” Pure Mathematics, Vol. 2, No. 4, 2012, pp. 192-201. doi:10.4236/PM.2012.24030

[9] W. H. Ji and B. L. Hei, "The Series of Reciprocals of Binomial Coefficients Constructing by Splitting Terms,” Pure Mathematics, 2013, Vol. 3, No. 1, p. 18.doi:10.12677/PM.2013.31005 